\title{
Loss Aversion Implied by a Risk-Based Questionnaire
}

\author{
JÜRGEN VANDENBROUCKe AND GOSIA FORTUNA
}

\begin{abstract}
JÜRGEN
VANDENBROUCKE

is expert general manager and head of innovation at KBC Asset Management in Brussels, Belgium, and a guest lecturer at the University of Antwerp in Antwerp, Belgium. jurgen.jv.vandenbroucke@kbc.be
\end{abstract}

\section{GOSIA ForTuna}

is an actuary and innovation manager at KBC Asset Management in Brussels, Belgium. malgorzata.fortuna@kbc.be
I is standard practice for financial institutions to formulate model portfolios or investment advice for a limited set of investor profiles. Individual investors are appointed the profile that best fits their balance between risk and reward, based on a number of standardized questions.

This approach potentially suffers from at least two shortcomings. First, questionnaires expect investors to predict their own future behavior. Nobel laureate Daniel Kahneman (2009) states that indeed "people are poor forecasters of their future emotions and future tastes." Second, questionnaires restrict to the balance between dispersion of outcomes and average return, so-called risk preferences of an investor. Behavioral finance, and notably prospect theory, convincingly argues that investors in addition differ in their concern about losing money.

Regardless of the dispersion of outcomes, as a classical quantification of risk, investors tend to treat gains and losses differently. In general, people are more sensitive to losses than they are to commensurate gains. We refer to Kahneman and Tversky (1979) or Tversky and Kahneman (1992) for the original research and Camerer (2005) for practical arguments.

In this article we do restrict ourselves to an existing questionnaire. However, we will derive a more reliable anticipation of future behavior by processing the questionnaire data differently. We add a behavioral-based differentiating factor to the current riskbased profile definition. By doing so, we addresses the second shortcoming of the standard approach. Bodnaruk and Simonov (2016) show how the attitude toward loss of institutional investors impacts their investment allocations, investment performances, and career opportunities. It is fair to assume that the attitude toward loss is a differentiating factor among retail investors as well.

The outcome of our approach is a twodimensional investor profile that combines risk and loss preferences. This compares with the two-dimensional approach toward individual psychology developed by Bailard, Biehl, and Kaiser (1986). Their model relates to all things in life, not only to investments, and defines personalities along two axes: level of confidence (from anxious to confident) and method of action (from careful to impetuous). Any combination describes a persona. Similarly in our approach, any combination of risk and loss preferences defines a homogeneous client group.

The remainder of the article is structured as follows. The first section overviews the existing questionnaire, which we use as a data source. The following section describes the "as is" procedure currently being used to assign risk-based profiles to retail investors. We obverse that some risk profiles capture the majority of clients, and hypothesize that 
as a result, investors that share the same risk profile may still behave very differently. A third section outlines how we validated this hypothesis through field research in collaboration with relationship managers. The fourth section motivates the "to be" processing procedure, which expands the existing risk profiles with subcategories that differ in the attitude toward loss; the fifth section shows how the model predictions match revealed investor behavior as observed by relationship managers. The final section summarizes our main conclusions.

\section{THE QUESTIONNAIRE}

The financial institution that participated in our project serves 11 million customers. More information on this financial institution can be found in the most recent annual report, which is available at www.kbc .com.

The institution currently applies a standard questionnaire for its investor profiling. We take the questionnaire as given and outline its structure in this section.

The questionnaire consists of three parts. Part I investigates the investor's financial situation and Part II asks about investment knowledge and experience. Part III is most relevant for our application, as it lists three questions that assess the investor's preferences. The three questions deal with 1) the investment horizon, 2) the trade-off between risk and reward, and 3) the alleged reaction to decreases in market value. The questions cover topics imposed by regulation. They have no specific theoretical or psychological underpinning.

Each of the three questions in Part III has four predefined answers from which investors can choose. We refer to the appendix for the exact formulation. The first answer to each question is the most conservative and the fourth answer is the most daring. Each answer is linked to a numerical value: 1 for answer 1, 3 for answer 2, 5 for answer 3, or 7 for answer 4 (see Exhibit 1).

\section{“AS IS": CLASSICAL RISK PROFILES}

This section describes the "as is" profiling procedure, that is, how the financial institution processes the questions and answers described in Exhibit 1 to come up with a risk profile for the investor.

The investor is appointed to one of four likely risk profiles based on the sum of the answer combination in Part 3 of the questionnaire: Sum $=$ horizon + risk + decrease. Exhibit 2 lists the four risk profiles and the
E X H I B I T 1

Part III's Three Questions, Each with Four Possible Answers, and Their Corresponding Values

\begin{tabular}{lc}
\hline $\begin{array}{l}\text { Question in Part 3 } \\
\text { of Questionnaire }\end{array}$ & $\begin{array}{c}\text { Four Possible } \\
\text { Answers }\end{array}$ \\
\hline Horizon & $1 / 3 / 5 / 7$ \\
Risk & $1 / 3 / 5 / 7$ \\
Decrease & $1 / 3 / 5 / 7$ \\
\hline
\end{tabular}

Notes: Note that part III of the questionnaire implies a total of $4^{3}=64$ answer combinations.

\section{E X H I B I T 2}

The Link between Part 3 of the Questionnaire and a Risk Profile

\begin{tabular}{lcc}
\hline Risk Profile & $\begin{array}{c}\text { Sum }=\text { Horizon }+ \\
\text { Risk }+ \text { Decrease }\end{array}$ & Occurence \\
\hline Very Defensive & Sum $<6$ & $4 / 64$ \\
Defensive & $6 \leq$ Sum $<12$ & $28 / 64$ \\
Dynamic & $12 \leq$ Sum $<18$ & $28 / 64$ \\
Very Dynamic & $18 \leq$ Sum & $4 / 64$
\end{tabular}

cut-off values that apply, and highlights the number of answer combinations out of the total of 64 that lead to the corresponding risk profile.

The investor's final risk profile can nevertheless deviate from the logic in Exhibit 2 for two main reasons: the investor has the right to simply scale down the outcome of the logic shown in Exhibit 2, and the financial institution also can overrule the outcome. The latter is the case whenever the information in Part 1 and/or Part 2 indicates that the investor is insufficiently wealthy or insufficiently skilled. If so, the investor can at most be classified as defensive.

In summary, four risk profiles are put forward, with the processing procedure favoring the middle two, because the defensive and dynamic profiles have a higher likelihood of occurring. The defensive or dynamic profiles are attained in 58 out of 64 answer combinations. In addition, the defensive profile has a low barrier to entry because a number of dynamic answer combinations can be expected to be rescaled to a defensive profile, either because the investor opted to downscale or because the financial institution overruled. This is effectively what we see in the data.

Exhibit 3 shows the distribution of a representative, anonymous sample of over 145,000 investors. From 


\section{E X H I B I T 3 \\ Statistics on Investor Data}

\begin{tabular}{|c|c|c|c|}
\hline \multicolumn{4}{|c|}{$\begin{array}{l}\text { Panel A: Number of linvestors } \\
\text { in Each of the Four Risk Profiles }\end{array}$} \\
\hline & & \# clients & $\%$ clients \\
\hline \multirow{4}{*}{ 晃 } & VDEF & 9275 & $6.4 \%$ \\
\hline & DEF & 73323 & $50.3 \%$ \\
\hline & DYN & 58043 & $39.8 \%$ \\
\hline & VDYN & 5230 & $3.6 \%$ \\
\hline
\end{tabular}

Panel B: Relation between the Official Risk Profile and the Numeric Value of the Answer Combination in Part 3 of the Questionnaire

\begin{tabular}{|c|c|c|c|c|c|}
\hline & \multicolumn{4}{|c|}{ Calculated In Part III } \\
\hline & & VDEF & DEF & DYN & VDYN \\
\hline \multirow{4}{*}{ 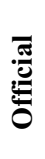 } & VDEF & $61.4 \%$ & $26.8 \%$ & $11.5 \%$ & $0.3 \%$ \\
\hline & DEF & $0 \%$ & $81.4 \%$ & $18.3 \%$ & $0.3 \%$ \\
\hline & DYN & $0 \%$ & $0 \%$ & $99.5 \%$ & $0.5 \%$ \\
\hline & VDYN & $0 \%$ & $0 \%$ & $0 \%$ & $100 \%$ \\
\hline
\end{tabular}

Notes: VDEF, DEF, DYN, and VDYN refer to the very defensive, defensive, dynamic, and very dynamic risk profile respectively. In Exhibit $3 b$ each row sums to $100 \%$. Each element refers to the percentage of investors within the risk profile indicated in the row head that gave an answer combination in Part 3 of the questionnaire that would justify a classification on the risk profile indicated in the column head. These numbers are based on a dataset of 145,871 investors for which we obtained anonymous data.

panel $3 \mathrm{a}$ it is clear that the defensive and dynamic risk profiles represent $90 \%$ of investors, with the defensive group being the largest.

Exhibit $3 \mathrm{~b}$ further displays all zeroes below the diagonal, which means no investor obtained a risk classification higher than the one based on Part 3 of the questionnaire. Numbers above the diagonal, however, are different from zero. Hence a substantial number of investors are classified in a more conservative risk profile than the one that would be justified based on the logic of Exhibit 2. Note particularly that more than $18 \%$ of investors with an official defensive risk profile actually provided a dynamic answer combination on the questions in Part 3.

The "as is" procedure obviously suffers from the critique of Faloon and Scherer (2017) that "scores are invented rather than derived from an academically cross-validated decision-making model." For legal and operational reasons, however, we preserve the existing risk-based profiling. We do not require all investors to be re-profiled; the idea is rather to add a second dimension to the existing investor profile, a differentiating factor that does have a sound methodological underpinning and is supported by the data. Readers may possibly enhance their current profiling procedure in a similar manner.

\section{FIELD RESEARCH}

This section reports on a collaboration with relationship managers to validate the idea of defining subgroups within existing risk profiles. The relationship managers meet their investors for a face-to-face portfolio screening at least twice per year, which means that the relationship manager knows the investor personally. The relationship managers received an introduction to the scope of the project during a brief conference call. We did not reveal the methodology that would be tested, only the motivation. We provided each relationship manager with a list of investors he or she services and revealed that all investors on the list qualify as defensive based on the existing profiling procedure.

During the field research we asked the relationship managers to rank each investor on a scale from 1 to 7 on the perceived attitude toward loss. How do these investors behave in real life? Each investor received one score, which was given by the relationship manager that services that investor in the local office. The score itself does not bear any particular meaning. What is important is the qualitative description, which ranges from "the investor sees interim losses as an inevitable part of investing and even considers them an opportunity" to "this investor reacts quickly and nervously to any decrease in portfolio value." Finally, the instructions that came with the exercise emphasized that the investment managers should not to consult the database and surely not involve any investor. The score should reflect the first idea that comes to the relationship manager's mind when envisioning the person who corresponds to the name on the list.

A total of 24 relationship managers scored 572 investors. We were curious to see whether the scores were dispersed or clustered, given that all investors belong to the same risk profile. Exhibit 4 shows the number of investors for each relationship manager who were given a score of 1 to 7 . 


\section{E X H I B I T 4}

572 Investor Scores Received from 24 Relationship Managers

\begin{tabular}{|c|c|c|c|c|c|c|c|c|}
\hline & & Score 1 & Score 2 & Score 3 & Score 4 & Score 5 & Score 6 & Score 7 \\
\hline \multirow{24}{*}{ 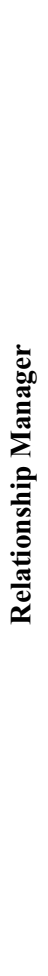 } & 1 & 2 & 1 & 0 & 7 & 8 & 3 & 4 \\
\hline & 2 & 3 & 4 & 1 & 7 & 6 & 4 & 0 \\
\hline & 3 & 8 & 1 & 0 & 1 & 5 & 8 & 1 \\
\hline & 4 & 8 & 6 & 2 & 6 & 2 & 1 & 0 \\
\hline & 5 & 0 & 2 & 6 & 5 & 9 & 0 & 0 \\
\hline & 6 & 0 & 0 & 5 & 8 & 12 & 2 & 2 \\
\hline & 7 & 5 & 4 & 3 & 4 & 3 & 2 & 2 \\
\hline & 8 & 4 & 1 & 2 & 4 & 4 & 1 & 8 \\
\hline & 9 & 2 & 0 & 1 & 4 & 4 & 6 & 1 \\
\hline & 10 & 2 & 5 & 0 & 9 & 5 & 2 & 0 \\
\hline & 11 & 3 & 2 & 4 & 0 & 3 & 4 & 3 \\
\hline & 12 & 9 & 1 & 2 & 6 & 2 & 6 & 0 \\
\hline & 13 & 0 & 2 & 2 & 4 & 7 & 6 & 1 \\
\hline & 14 & 1 & 4 & 4 & 4 & 8 & 2 & 1 \\
\hline & 15 & 2 & 11 & 5 & 5 & 3 & 1 & 0 \\
\hline & 16 & 5 & 4 & 6 & 1 & 4 & 3 & 0 \\
\hline & 17 & 6 & 2 & 0 & 2 & 3 & 5 & 4 \\
\hline & 18 & 2 & 2 & 5 & 4 & 10 & 1 & 0 \\
\hline & 19 & 3 & 3 & 7 & 9 & 5 & 0 & 0 \\
\hline & 20 & 2 & 1 & 5 & 8 & 6 & 3 & 1 \\
\hline & 21 & 2 & 2 & 3 & 3 & 4 & 6 & 5 \\
\hline & 22 & 1 & 5 & 6 & 3 & 3 & 1 & 4 \\
\hline & 23 & 3 & 3 & 5 & 11 & 4 & 0 & 0 \\
\hline & 24 & 2 & 0 & 1 & 3 & 4 & 7 & 3 \\
\hline
\end{tabular}

Notes: For each relationship manager, the exhibit shows the number of investors who were given each score. The score reflects the relationship manager's assessment of their investors' attitude toward loss. A higher score indicates a higher tolerance for loss.

Exhibit 4 reveals that scores at the level of the individual relationship manager are dispersed, which validates our research question; the existing risk profiles do not lead to homogeneous groups, notably regarding attitude to loss. Exhibit 5 aggregates the scores over all relationship managers.

In general, relationship managers confirm that even investors who share the same risk profile do not all react to loss the same way. The scores populate the entire spectrum that was provided.

The challenge now is to anticipate the ex-post perceived investor behavior by defining ex-ante clusters based on available investor data.

\section{“TO BE": BEHAVIORAL INVESTOR PROFILES}

The "to be" procedure identifies subgroups among investors that share the same classical risk profile.
The subgroups differ in their attitude toward loss. In this section we motivate a simple procedure to define such subgroups based on available information.

\section{Motivation}

The previous section introduced the structure of the questionnaire. Diving deeper into the data, Exhibit 6 reports the dispersion of the answers to each of the questions in Part 3. The tables of Exhibit 6 contain percentages that sum to $100 \%$ per risk profile (row), and each percentage is the proportion of investors with that risk profile whose answer is represented by the value in the column header.

Consider the question related to risk. Dynamic investors, for example, are very likely to give a dynamic answer on the risk question. For other profiles as well the answer to the risk question largely matches the 


\section{E X H I B I T 5}

\section{The 572 Investor Scores Aggregated over 24 Relationship Managers}

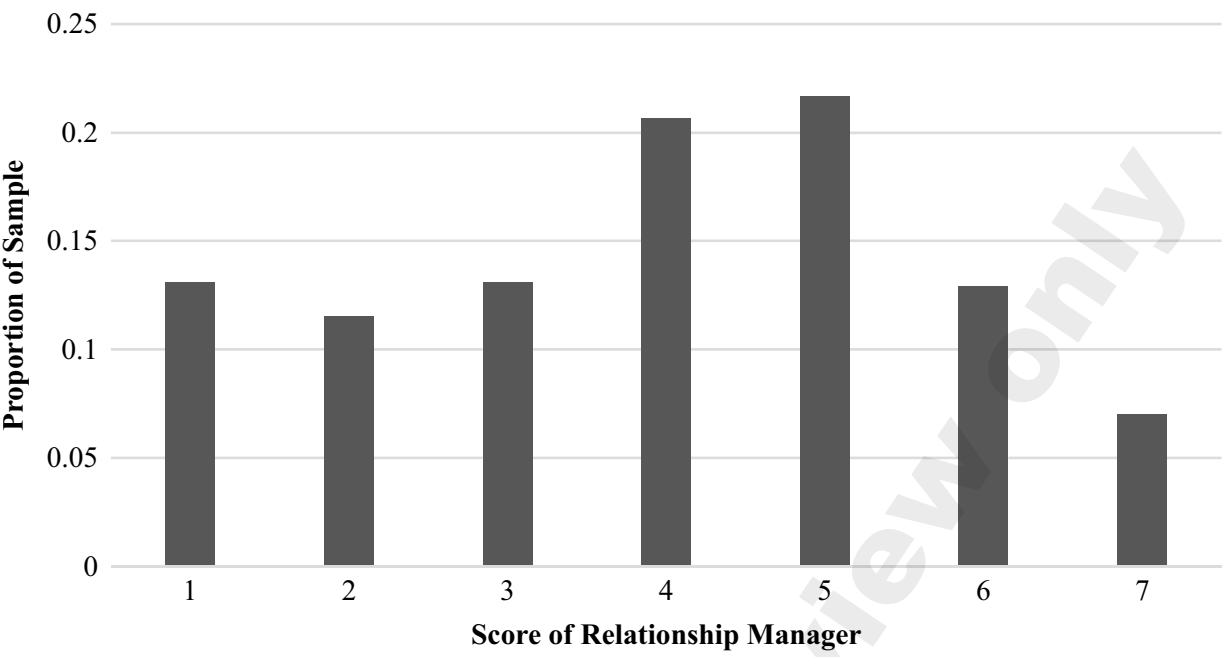

naïve prediction one would make for that profile. All percentages on the diagonal are high and close to $80 \%$. This is not surprising, as the questionnaire results in a risk profile, after all. Our definition of subgroups within risk profiles will therefore include the answers on horizon and decrease, because the risk question clearly relates to risk preferences.

The link between investment horizon, loss aversion, and the (un)willingness to hold investments traces back to the behavioral economics research of Benardzi and Thaler (1995). Exhibit 6 implies that horizon is indeed a source of diversity within profiles. Most percentages on the diagonal do not exceed $50 \%$, which means that the investor's horizon is not a reliable indicator of the risk profile. Whether the diversity in horizon links to loss attitude remains to be tested, of course.

The answers on the decrease question are again more concentrated around the investor's risk profile. However, the question clearly focuses on downside risk, which naturally links to loss attitude and will therefore be included in the definition of loss aversion subgroups.

We finally add the age of the investor as a differentiating factor. When looking for determinants of loss aversion, there is one common element in the research: loss tolerance decreases with age (see, for example, Johnson et al. [2006], Gächter et al. [2007], Hjorth and Fosgerau [2011], and Arora and Kumari [2015]).

\section{E X H I B I T 6}

\section{Statistics on the Answers in Part 3 of the} Questionnaire

\begin{tabular}{|c|c|c|c|c|c|}
\hline 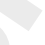 & & 1 & 3 & 5 & 7 \\
\hline \multicolumn{6}{|c|}{ Answer on Question Related to Horizon } \\
\hline \multirow{4}{*}{$\frac{\bar{\pi}}{\frac{\pi}{0}}$} & VDEF & $48.2 \%$ & $27.2 \%$ & $9.0 \%$ & $15.5 \%$ \\
\hline & DEF & $11.3 \%$ & $45.5 \%$ & $25.9 \%$ & $17.3 \%$ \\
\hline & DYN & $0.3 \%$ & $18.7 \%$ & $48.9 \%$ & $32.1 \%$ \\
\hline & VDYN & $0 \%$ & $0 \%$ & $17.6 \%$ & $82.4 \%$ \\
\hline \multicolumn{6}{|c|}{ Answer on Question Related to Risk } \\
\hline \multirow{4}{*}{ } & VDEF & $71.2 \%$ & $24.2 \%$ & $4.3 \%$ & $0.3 \%$ \\
\hline & DEF & $9.2 \%$ & $79.6 \%$ & $10.8 \%$ & $0.4 \%$ \\
\hline & DYN & $0.1 \%$ & $15.2 \%$ & $82.8 \%$ & $1.9 \%$ \\
\hline & VDYN & $0 \%$ & $0 \%$ & $19.0 \%$ & $81.0 \%$ \\
\hline & \multicolumn{5}{|c|}{ Answer on Question Related to Decrease } \\
\hline \multirow{4}{*}{$\frac{\bar{\pi}}{\stackrel{\frac{\pi}{2}}{0}}$} & VDEF & $50.4 \%$ & $41.9 \%$ & $7.4 \%$ & $0.3 \%$ \\
\hline & DEF & $1.0 \%$ & $78.3 \%$ & $20.3 \%$ & $0.4 \%$ \\
\hline & DYN & $0 \%$ & $15.8 \%$ & $81.3 \%$ & $2.9 \%$ \\
\hline & VDYN & $0 \%$ & $0 \%$ & $23.9 \%$ & $76.1 \%$ \\
\hline
\end{tabular}

Note: Similar to Exhibit 3, these numbers are based on a dataset of 145,871 investors for which we obtained anonymous data.

\section{Defining a New Variable}

We now apply the three input parametershorizon, decrease, and age - to calculate a new variable. We define the variable such that a higher value assumes a 


\section{E X H I B I T 7 \\ Distribution of the Calculated Variable Horizon*Decrease/Age}

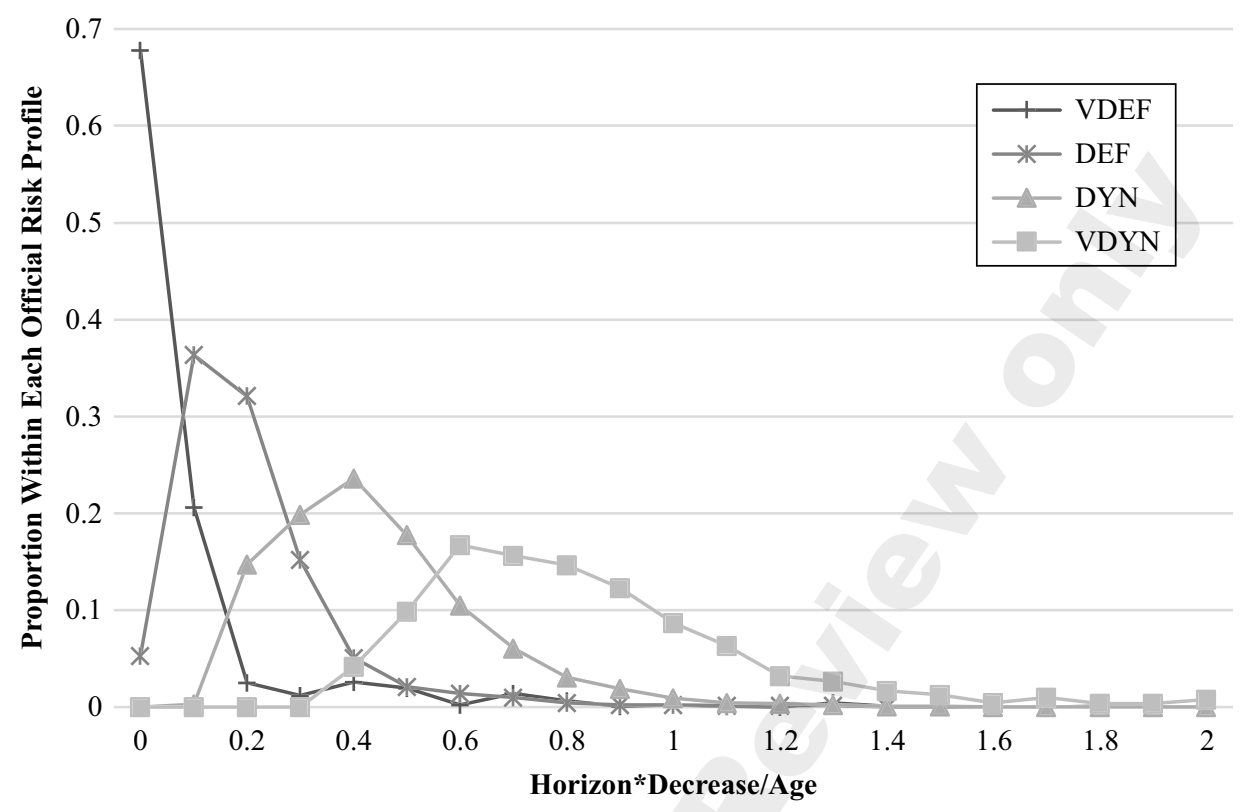

Note: These distributions are based on the selection of 49,421 investors for which the dataset contains the investor's age.

higher loss tolerance. In line with the hypotheses above, the variable will take a higher value if the investor claims to have a longer investment horizon, and/or claims be more tolerant for interim value decreases, and/or is of a younger age. Rather than summing the numeric values of the horizon and decrease answers, as when deriving the risk profile, we choose to multiply the values, because this spreads the outcome of the likely combinations over a wider range. The product of the answers on horizon and decrease is then divided by the age of the investor to obtain a new variable with the desired sensitivities: horizon*decrease/age. Exhibit 7 shows the distribution of the new variable per risk profile.

The shape of the distribution per risk profile is determined by the dispersion of the horizon answer (among others). Exhibit 6 explains why the curves in Exhibit 7 are positioned more to the right for more dynamic risk profiles. However, the current variable is more refined. First, the horizon answer is magnified or tempered depending on whether the investor answers the decrease question more dynamically. Second, any given answer combination on horizon and decrease is adjusted for the age of the investor. Put differently, if a younger and an older investor prefer the same horizon and give the same answer to the decrease question, the older investor will be assumed to be less tolerant for loss compared to the younger investor. These sensitivities of the new variable are deemed intuitive and will be tested.

\section{Defining Subgroups}

We next describe the procedure to define subgroups or clusters within a given risk profile, based on the variable horizon*decrease/age. The procedure takes the defensive profile as an example because this risk profile is the most populated and the focus of our field research. First, we calculate the variable horizon*decrease/age for the "population," i.e., all available defensive investors.

Second, we assume three likely positions on the dimension that defines attitude toward loss. Note that this is a modeling choice; we could have assumed more clusters. Cluster 1 is defined as $p_{i} \leq P_{1}$ with $\operatorname{Prob}\left(p_{i} \leq P_{1}\right)=\alpha$. Cluster 3 is defined as $p_{i}>P_{2}$ with $\operatorname{Prob}\left(p_{i}>P_{2}\right)=\alpha$. Cluster 2 is logically defined as $P_{1}<p_{i} \leq P_{2}$. As such, we use the distribution of the variable $p_{i}$ to define two cut-off levels, $P_{1}$ and $P_{2}$. We impose some structure by assuming that investors with the least tolerance for loss (Cluster 1) and investors with the most tolerance for loss 
E X H I B I T 8

Cut-off Values to Defined Clusters of Defensive Investors

\begin{tabular}{lccccccc}
\hline & $\boldsymbol{\alpha}=\mathbf{5 \%}$ & $\boldsymbol{\alpha}=\mathbf{1 0} \%$ & $\boldsymbol{\alpha}=\mathbf{1 5 \%}$ & $\boldsymbol{\alpha}=\mathbf{2 0 \%}$ & $\boldsymbol{\alpha}=\mathbf{2 5 \%}$ & $\boldsymbol{\alpha}=\mathbf{3 0 \%}$ & $\boldsymbol{\alpha}=\mathbf{3 5 \%}$ \\
\hline P1 & 0.0492 & 0.0893 & 0.1059 & 0.1139 & 0.1200 & 0.1286 & 0.1385 \\
P2 & 0.4839 & 0.3684 & 0.3125 & 0.2830 & 0.2586 & 0.2381 & 0.2206 \\
\hline
\end{tabular}

Note: The table contains the cut-off values for the variable horizon*decrease/age that define clusters for defensive investors.

\section{E X H I B I T 9}

Statistics for Clusters of Defensive Investors

\begin{tabular}{|c|c|c|c|c|c|c|c|c|}
\hline & & $\alpha=5 \%$ & $\alpha=10 \%$ & $\alpha=15 \%$ & $\alpha=20 \%$ & $\alpha=25 \%$ & $\alpha=30 \%$ & $\alpha=35 \%$ \\
\hline \multirow{5}{*}{ 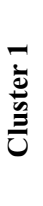 } & Avg Horizon & 1.27 & 1.43 & 1.89 & 2.18 & 2.31 & 2.42 & 2.50 \\
\hline & Avg Risk & 3.10 & 3.10 & 3.07 & 3.06 & 3.05 & 3.04 & 3.05 \\
\hline & Avg Decrease & 2.72 & 3.03 & 3.06 & 3.05 & 3.05 & 3.04 & 3.04 \\
\hline & Part III > 11 & $0 \%$ & $0 \%$ & $0 \%$ & $0 \%$ & $0 \%$ & $0 \%$ & $0 \%$ \\
\hline & Avg Age & 76 & 68 & 72 & 74 & 74 & 73 & 72 \\
\hline \multirow{5}{*}{ 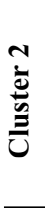 } & Avg Horizon & 3.57 & 3.63 & 3.62 & 3.62 & 3.60 & 3.58 & 3.57 \\
\hline & Avg Risk & 3.05 & 3.04 & 3.04 & 3.05 & 3.06 & 3.07 & 3.08 \\
\hline & Avg Decrease & 3.28 & 3.25 & 3.24 & 3.24 & 3.24 & 3.24 & 3.24 \\
\hline & Part III > 11 & $5 \%$ & $3 \%$ & $2 \%$ & $2 \%$ & $1 \%$ & $1 \%$ & $1 \%$ \\
\hline & Avg Age & 62 & 64 & 64 & 64 & 64 & 63 & 63 \\
\hline \multirow{5}{*}{ 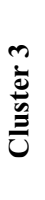 } & Avg Horizon & 5.40 & 4.95 & 4.83 & 4.75 & 4.68 & 4.63 & 4.57 \\
\hline & Avg Risk & 3.69 & 3.43 & 3.31 & 3.24 & 3.19 & 3.16 & 3.14 \\
\hline & Avg Decrease & 4.38 & 4.02 & 3.87 & 3.78 & 3.72 & 3.67 & 3.64 \\
\hline & Part III > 11 & $68 \%$ & $51 \%$ & $42 \%$ & $35 \%$ & $29 \%$ & $25 \%$ & $22 \%$ \\
\hline & Avg Age & 33 & 36 & 40 & 42 & 45 & 47 & 49 \\
\hline
\end{tabular}

(Cluster 3) represent a same portion $\alpha$ of the population. We will address the sensitivity of our results with respect to the choice for $\alpha$. Exhibit 8 reports the resulting cutoff values, for $\alpha$ ranging from 5\% to 35\%.

Exhibit 9 reports statistics for clusters based on the cut-off values in Exhibit 8.

Obviously, the clusters in Exhibit 9 differ in terms of average horizon, decrease, and age in a way that is imposed by the model. The most loss averse, Cluster 1 , reports, for any value of $\alpha$, a lower average answer on horizon, a lower average answer on decrease and a higher average age compared to Cluster 2 or Cluster 3. The next section investigates whether these differences align with observed investor behavior from the field research.

We conclude this section with an interesting final observation in Exhibit 9, which is that the most loss tolerant cluster, Cluster 3, contains virtually all defensive investors that provided a dynamic answer combination in the questionnaire. Consider the column with $\alpha=15 \%$. Using this value for $\alpha, 41.9 \%$ of the defensive investors in Cluster 3 gave an answer combination in Part 3 of the questionnaire that adds up to more than 11 (cfr. Exhibit 2 ). If Part 3 of the questionnaire were the only criterion, these investors would be considered dynamic.

The methodology captures those investors in the most loss tolerant cluster within their official, defensive, risk profile. Put differently, if an investor provides an answer combination where horizon + risk + decrease exceeds 11 but nevertheless qualifies as a defensive investor, than most likely horizon*decrease/age exceeds the threshold to belong to the loss tolerant cluster. As a consequence, Cluster 2 only contains $2.2 \%$ defensive investors with a dynamic answer combination in Part 3 of the questionnaire. No such investors are considered most loss averse, for any value of $\alpha$. Yet, Cluster 3 groups more than just these "special" defensive investors. Cluster 3 also contains investors with a defensive answer 


\section{E X H I B I T 10}

\section{Scores of Defensive Investors, Clustered for Behavioral Investor Profile}

\begin{tabular}{|c|c|c|c|c|c|c|c|c|}
\hline & & $\alpha=5 \%$ & $\alpha=10 \%$ & $\alpha=15 \%$ & $\alpha=20 \%$ & $\alpha=25 \%$ & $\alpha=30 \%$ & $\alpha=35 \%$ \\
\hline \multirow{2}{*}{$=$} & \# Investors & 41 & 65 & 86 & 121 & 161 & 196 & 249 \\
\hline & Avg RM Score & 3.29 & 3.49 & 3.65 & 3.77 & 3.73 & 3.77 & 3.80 \\
\hline \multirow{2}{*}{ 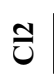 } & \# Investors & 523 & 476 & 429 & 370 & 308 & 247 & 171 \\
\hline & Avg RM Score & 3.96 & 3.97 & 3.97 & 3.96 & 4.01 & 3.98 & 3.99 \\
\hline \multirow{6}{*}{$\stackrel{m}{u}$} & \# Investors & 8 & 31 & 57 & 81 & 103 & 129 & 152 \\
\hline & Avg RM Score & 5.13 & 4.19 & 4.02 & 4.02 & 4.01 & 4.08 & 4.08 \\
\hline & Significance & & & & & & & \\
\hline & Cluster 3 vs 1 & $1 \%$ & $5 \%$ & $15 \%$ & $20 \%$ & $15 \%$ & $10 \%$ & $10 \%$ \\
\hline & Cluster 2 vs 1 & $1 \%$ & $5 \%$ & $10 \%$ & $15 \%$ & $10 \%$ & $15 \%$ & $15 \%$ \\
\hline & Cluster 3 vs 2 & $5 \%$ & - & - & - & - & - & - \\
\hline
\end{tabular}

Notes: For each of the three clusters, the table lists the number of investors that belong to the cluster and the average score of the relationship managers. The lower panel contains a test of means on the average scores of the relationship managers between clusters, given the value for $\alpha$. The panel shows the tightest significance level that rejects the null hypothesis of equal average scores, where "-" means the significance level exceeds $20 \%$.

in Part 3 of the questionnaire, i.e., where horizon + risk + decrease does not exceed 11 . The clustering ultimately depends on the value of the variable horizon*decrease/ age. The final section reports how the model-based clustering relates to perceived investor behavior.

\section{FIELD VALIDATION}

In this section we assess whether the heterogeneity among defensive investors, as reported by their relationship managers in Exhibits 4 and 5, can be linked to the variable horizon*decrease/age. Exhibit 10 reports the main results. The 572 defensive investors of the project are divided into three clusters using cut-off values for the variable horizon*decrease/age as listed in Exhibit 8.

According to Exhibit 10, the average score of the relationship managers is lowest for investors in Cluster 1 and highest in Cluster 3. Put differently, ranking defensive investors based on the variable horizon*decrease/ age highlights investors' diversity that aligns with their loss attitude as observed by relationship managers. A comparison of the scores in Clusters 1 and 2 yields the same conclusion regardless of the value for $\alpha$. Based on the significance levels, we observe that Clusters 2 and 3 are the least different. However, we previously noted that Cluster 3 captures virtually all, but not only, defensive investors with a dynamic answer combination in the questionnaire. This conclusion also holds for the 572 defensive investors of the project (not shown here). We believe that such a qualitative difference gives the compliance and legal department a reason to consider Cluster 3 indeed different from Cluster 2, despite the lack of quantitative significance.

Exhibit 10 also implicitly validates the sensitivities embedded in our methodology. Given the attitude toward risk, we find that loss tolerance is a differentiating feature in investor behavior. Given the attitude toward risk, loss tolerance is found to increase as a function of the alleged investment horizon and the alleged attitude toward decreases, while loss tolerance is found to erode with age.

We performed a comparable statistical analysis in case investors are clustered on the basis of unprocessed questionnaire data, such as the answer on horizon or decrease. The scores of the relationship manager do not align with such crude usage of the questionnaire data. The variable horizon*decrease/age does a far better job in anticipating investor behavior based on available investor information. This confirms that the proposed methodology is more than a simple increase of the number of standard risk profiles. The results identify risk and loss preferences as complementary aspects of investor behavior, hence the representation as loss aversion subgroups within existing risk profiles.

\section{CONCLUSION AND IMPLICATIONS}

We started from the reflection that a limited set of risk-based investor profiles is unlikely to be successful in capturing investor diversity. The literature on behavioral 
finance motivated us to also include emotional elements, such as attitude toward loss.

The behavioral profile we propose builds on the structure of a standard risk-based procedure. The assessment of an investor's risk profile is left unchanged. We rather add a dimension that relates to the attitude toward loss. All is based on existing information. We showed that relevant heterogeneity surfaces, validated by a field experiment.

In the wording of Nobel laureate Richard Thaler, we tend to call this enhancement of the classical risk profile a "nudge": a supposedly irrelevant factor that influences choices in a way "that will make choosers better off, as judged by themselves" (see Thaler [2015, p. 325]). The latter is confirmed by a field experiment. The model-based anticipation on investor loss aversion matches the recordings of the investor's relationship manager.

One immediate implication of the enhanced investor profile is a more accurate description of investor preferences, which serves multiple strategic and commercial purposes. First and foremost, the enhanced investor profile makes it possible to customize financial services. Pre- or post-sales procedures like onboarding or reporting can be made more personal and customercentric. Second, the financial institution might want to take investor preferences into account when defining its marketing and communications policy. For example, the hit ratio of commercial communication can be expected to improve if such communication is aligned in wording and frequency with a deeper understanding of investor preferences. Finally, a richer model of investor preferences contributes to the scalability and conversion rates of digital advisory services by making an offer to which people can relate.

\section{A P P E N D I X}

\section{PART 3 OF THE QUESTIONNAIRE}

Part 3 of the questionnaire polls how the investor looks at investing and contains three questions, each with four predefined answers.

The first question asks for the investor's investment horizon. The investor is offered four possible answers: "less than 3 years," "between 3 and 6 years," "between 6 and 10 years," or "beyond 10 years."
The second question aims to assess the investor's view on the trade-off between risk and reward. The investor can again choose between four likely answers: "stability is the most important," "taking on some more risk is fine but most of my investments should be considered safe," "I realize that taking on more risk is required to target a higher return," and "I aim for the highest likely return in the long run."

The third question asks how investors think they would react should the value of their investment decreases, possibly more than initially anticipated. The four possible answers are: "this is a reason to sell my positions," "this is a trigger to consult my advisor," "this is considered only temporary," and "this is considered to be an opportunity."

\section{ACKNOWLEDGMENTS}

We would like to thank Peter Bauwens, Laurens Swinkels, and Andries Verreet, who made many helpful remarks on previous drafts while Alison Musgrave and Gavin Curran substantially improved the readability. We also thank Joris De Moor for providing details on the "as is" profiling procedure, Enrico DeGiorgi for sharing his view on the validation of the "to be" profiling process, and Chris Sterckx for his continuing support.

\section{REFERENCES}

Arora, M., and S. Kumari. 2015. "Risk Taking in Financial Decisions as a Function of Age, Gender: Mediating Role of Loss Aversion and Regret." International Journal of Applied Psychology 5 (4): 83-89.

Bailard, T., D. Biehl, and R. Kaiser. Personal Money Management. Chicago, IL: Science Research Associates (1986).

Benardzi, S., and R. Thaler. 1995. "Myopic Loss Aversion and the Equity Premium Puzzle." The Quarterly Journal of Economics 110 (1): 73-92.

Bodnaruk, A., and A. Simonov. 2016. "Loss-averse Preferences, Performance, and Career Success of Institutional Investors." Review of Financial Studies 29 (11): 3140-3176.

Camerer, C. 2005. "Three Cheers-Psychological Theoretical, Empirical-For Loss Aversion.” Journal of Marketing Research 42 (2): 129-133.

Faloon, M., and B. Scherer. 2017. "Individualization of Robo-advice." The Journal of Wealth Management 20 (1): 30-36. 
Gächter, S., E. Johnson, and A. Herrmann. 2007. "Individuallevel Loss Aversion in Riskless and Risky Choices." Center for Decision Research and Experimental Economics. Discussion Paper 2007-02.

Hjorth, K., and M. Fosgerau. 2011. "Loss Aversion and Individual Characteristics." Environmental and Resource Economics 49 (4): 573-596.

Johnson, E., S. Gächter, and A. Herrmann. 2006. "Exploring the Nature of Loss Aversion." Institute for the Study of Labor. Discussion paper 2015.

Kahneman, D. “The Myth of Risk Attitudes.” 2009. The Journal of Portfolio Management 36 (1): 1.

Kahneman, D., and A. Tversky. 1979. "Prospect Theory: An Analysis of Decision under Risk. Econometrica 47 (2): 263-291.

Thaler, R. Misbehaving-The Making of Behavioral Economics. New York, NY: W.W. Norton \& Company (2015): 432.

Tversky, A., and D. Kahneman. 1992. "Advances in Prospect Theory: Cumulative Representation of Uncertainty." Journal of Risk and Uncertainty 5: 297-323.

To order reprints of this article, please contact David Rowe at d.rowe@pageantmedia.com or 646-891-2157. 